\title{
Anesthetic considerations for awake craniotomy
}

\section{Seung Hyun Kim and Seung Ho Choi}

Received June 5, 2020

Accepted June 17, 2020

\section{Corresponding author}

Seung Ho Choi, M.D., Ph.D.

Department of Anesthesiology and

Pain Medicine, Anesthesia and Pain

Research Institute, Yonsei University

College of Medicine, 50-1 Yonsei-ro,

Seodaemun-gu, Seoul 03772, Korea

Tel: 82-2-2228-2415

Fax: 82-2-2227-7897

E-mail: csho99@yuhs.ac
Department of Anesthesiology and Pain Medicine, Anesthesia and Pain Research Institute, Yonsei University College of Medicine, Seoul, Korea

Awake craniotomy is a gold standard of care for resection of brain tumors located within or close to the eloquent areas. Both asleep-awake-asleep technique and monitored anesthesia care have been used effectively for awake craniotomy and the choice of optimal anesthetic approach is primarily based on the preferences of the anesthesiologist and surgical team. Propofol, remifentanil, dexmedetomidine, and scalp nerve block provide the reliable conditions for intraoperative brain mapping. Appropriate patient selection, adequate perioperative psychological support, and proper anesthetic management for individual patients in each stage of surgery are crucial for procedural safety, success, and patient satisfaction.

Keywords: Awake craniotomy; Brain neoplasms; Conscious sedation; Craniotomy.

\section{INTRODUCTION}

Awake craniotomy has been adopted for the surgical treatment of intractable epilepsy more than 100 years ago, and is now considered as a gold standard of care for resection of tumors, such as primarily gliomas, located within or close to the eloquent areas (i.e., sensorimotor or language areas) of the brain $[1,2]$. The modern awake craniotomy techniques have evolved in combination with intraoperative neurophysiologic monitoring to identify the eloquent areas of the brain. An optimal tumor resection is the maximal removal of mass without any significant neurological deficit, such as motor or language function damage, for most brain tumors. The cortical mapping during awake craniotomy enables the identification of cortical and subcortical networks for individual patients' neurological functions. Compared with craniotomy under general anesthesia, awake craniotomy can provide wider extent of tumor removal without postoperative neurologic deficits and improved survival rates of patients. Therefore, it is now considered as the treatment of choice for surgery of brain tumor in the eloquent areas $[3,4]$. Smooth intraoperative emergence and adequate communi- cation between the surgeons and patients are important anesthetic considerations in this surgery, and are the key factors for successful awake craniotomy.

\section{ANESTHETIC ADVANTAGES OF AWAKE CRANIOTOMY}

Awake craniotomy has several anesthetic benefits and surgical advantages. Patients undergoing awake craniotomy can avoid general anesthesia-related procedures, such as endotracheal intubation and mechanical ventilation. General anesthesia-related hemodynamic and physiologic disturbances are reduced, and postoperative pain, nausea, and vomiting are reduced in awake craniotomy compared with the craniotomy under general anesthesia $[5,6]$. The surgical and anesthetic advantages of awake craniotomy are listed in Table 1.

Generally, inhalational anesthetic agents are rarely used in awake craniotomy, unless endotracheal intubation or insertion of laryngeal mask airway are performed, and only small dose of intravenous agents, such as propofol, is used [7]. Analgesia during awake craniotomy is mainly achieved

This is an Open Access article distributed under the terms of the Creative Commons Attribution Non-Commercial License (http://creativecommons.org/licenses/by-nc/4.0) which permits unrestricted non-commercial use, distribution, and reproduction in any medium, provided the original work is properly cited.

Copyright (C) the Korean Society of Anesthesiologists, 2020 
by the scalp nerve block with local anesthetic agents. Therefore, hypotension associated with general anesthesia is uncommon during awake craniotomy and subsequently, vasopressors are used less than that during general anesthesia [8]. Hence, patient's hemodynamic and physiologic status can be more stable in awake craniotomy than that in general anesthesia.

Possible adverse effect of general anesthetic agents, including inhalation anesthetic agents and opioids on cancer prognosis, such as increased recurrence or metastasis after the surgery, has been reported recently $[9,10]$. In contrast, local anesthetics can directly inhibit some cancer cells even though this effect seems agent specific [11]. However, the impact of avoiding general anesthesia during awake craniotomy on brain tumor prognosis has not been evaluated in prospective, randomized, and controlled trials.

Awake craniotomy is also associated with shorter hospital stay compared with the craniotomy under general anesthesia [12], potentially reducing the risks of hospital-acquired infection and deep vein thrombosis [13].

\section{PREOPERATIVE PREPARATION}

Awake craniotomy requires a highly cooperative patient and an expert surgical team. Appropriate patient selection and preparation are essential for the success of awake craniotomy. Preoperative airway evaluation should be performed in all patients. Although a patient with a difficult airway or

Table 1. Advantages of Awake Craniotomy

Surgical aspects
Better preservation of motor \& speech function
Shorter hospitalization
Reduced postoperative neurologic deficits
Improved survival
Anesthetic aspects
Less physiological disturbance
Avoidance of mechanical ventilation
Avoidance the adverse impact on immunity associated with general
anesthesia

obstructive sleep apnea is a poor candidate for awake craniotomy, but only the patient's refusal is considered as an absolute contraindication for awake craniotomy [6].

Control of preoperative anxiety before awake craniotomy is important and can be relieved by proper preoperative counseling about the anesthetic and surgical procedures. Therefore, preoperative consultation by anesthesiologist is an important process $[14,15]$. The anesthesiologist should outline the overall awake craniotomy procedures including the positioning, scalp nerve block, the possible discomfort, and the motor and language test. A good anesthesiologist-patient relationship is essential and the anesthesiologist should attempt to alleviate anxiety and discomfort of the patient as much as possible to ensure the success of awake craniotomy [15].

\section{ANESTHETIC APPROACHES FOR AWAKE CRANIOTOMY: OVERVIEW}

Various anesthetic techniques may be useful for awake craniotomy. Among them, there are two commonly used anesthetic methods for awake craniotomy: monitored anesthesia care (MAC) and asleep-awake-asleep (AAA) technique [4-6]. The anesthesiologists should provide sufficient sedation and analgesia during the initial craniotomy, a rapid and smooth emergence of patients is required for intraoperative neurophysiologic test including motor and language test, and brain mapping. After the tumor resection, sedation is often sufficient until completion of the surgery. The overall stages of awake craniotomy and anesthetic techniques are presented in Table 2. The sedation profile during the first stage of awake craniotomy, from scalp incision to dura opening, plays a pivotal role in the quality of intraoperative consciousness. The anesthesiologist should restore the consciousness of patient back to the preoperative state for neurophysiologic tests and brain mapping to be performed successfully.

In the MAC technique, adequate depth of sedation and

Table 2. The Stages of Awake Craniotomy and Anesthetic Options

\begin{tabular}{lccc}
\hline \multicolumn{1}{c}{ Stages } & Pre-awake stage & Awake stage & Post-awake stage \\
\hline Surgical procedure & Craniotomy & Neurophysiologic monitoring & Closure \\
& & Brain mapping \\
Tumor resection & \\
Anesthetic methods & GA (ETT or LMA) & Awake & GA or MAC \\
AAA & MAC & Awake & MAC \\
\hline
\end{tabular}

AAA: asleep-awake-asleep, GA: general anesthesia, ETT: endotracheal tube, LMA: laryngeal mask airway, MAC: monitored anesthesia care. 
spontaneous ventilation are maintained using low doses of sedative agents to avoid a sudden recovery from asleep state, which could be a risk factor for delirium on emergence and decrease neurophysiologic tests or brain mapping reliability. In contrast, the AAA technique uses higher doses of sedative agents. The target depth of AAA technique ranges from deep sedation to general anesthesia using mechanical ventilation, commonly by a laryngeal mask airway. The benefits of this technique as compared to the MAC technique are better comfort and pain control for the patient, and securing the patient's airway during the first stage of awake craniotomy. This approach is also advantageous for controlling brain swelling via hyperventilation and prevention of accidental patient movements.

Although there are significant differences in these two techniques, both the MAC and AAA techniques can be performed successfully in awake craniotomy and are found to be safe [16]. In a recent retrospective analysis comparing the MAC and AAA technique for awake craniotomy in a single center, both the techniques were equally effective and safe, and produced comparable perioperative outcomes, with a shorter duration of surgery in the MAC technique [17]. In another retrospective analysis comparing the effectiveness of the MAC and AAA techniques in 64 patients undergoing supratentorial brain tumor resection, the incidence of seizures and agitation were higher in the MAC group than that in the AAA group. However, blood pressure was higher and more antihypertensive treatment was required in the AAA group than that in the MAC group during the neurophysiologic examination [18]. Therefore, with a good cooperation among anesthesiologists, surgeons, and neurophysiologists, the MAC technique can be performed successfully with similar safety and comfort for the patients compared to the AAA technique [19].

\section{ANESTHETIC APPROACHES FOR AWAKE CRANIOTOMY: SEDATIVE COMPONENTS}

A combination of propofol and remifentanil has been considered as the standard protocol for sedation during the first stage of awake craniotomy because of the ease of use and reliability $[6,20]$. The propofol and remifentanil-based AAA technique allows a smooth emergence and rapid recovery of consciousness for intraoperative neurophysiologic testing [21]. The main disadvantage of this approach is the potential risk of delirium on emergence before intraoperative neurophysiologic testing and brain mapping [6]. The propofol and remifentanil-based MAC technique is associated with dose-dependent respiratory depression, which can produce hypercapnia and subsequent brain edema. Therefore, achieving the optimal sedation level for an individual is crucial. Meanwhile, light sedation has the risk of causing accidental patient movement and anxiety is likely. Generally, state of drowsiness but readily responsive is considered as the optimal sedation in awake craniotomy [6], and experienced anesthesiologists are required to accomplish this balance in the complex setting of awake craniotomy. The electroencephalography-derived monitors, such as the electroencephalogram-derived bispectral index, and target-controlled infusion of propofol and remifentanil can be useful for this setting. The Schneider model is recommended for the target-controlled infusion of propofol in awake craniotomy for maintaining patients' spontaneous ventilation [22].

Dexmedetomidine an alternative to propofol for the MAC technique during awake craniotomy can also be used. It is a selective alpha-2 agonist with sedative, analgesic, anxiolytic, and sympatholytic properties. The advantageous effects of dexmedetomidine, such as minimal effect on neurophysiologic monitoring, stable hemodynamics, and minimal respiratory depression, makes it suitable for sedation during awake craniotomy [23]. In a recent study comparing propofol-remifentanil and dexmedetomidine during awake craniotomy in patients undergoing supratentorial tumor resection, dexmedetomidine provided comparable surgical environment in terms of quality of intraoperative brain mapping and efficacy of sedation, with less respiratory adverse events compared with the propofol-remifentanil group [24]. Similarly, a retrospective cohort study including 55 patients demonstrated that dexmedetomidine and the scalp nerve block were used successfully in awake craniotomy without any urgent airway intervention or unplanned conversion to general anesthesia [25]. Although high dose of dexmedetomidine may cause bradycardia, significant inter-individual variability of the sedative state, and a prolonged recovery from sedation after the cessation of infusion, such a high dose is not required during awake craniotomy. Moreover, it is especially useful for high-risk patients or when prolonged duration of surgery is anticipated [26].

Inhalational anesthetics, such as sevoflurane, are also used for the AAA technique in awake craniotomy. However, these agents have the potential to increase intracranial pressure and induce nausea and vomiting during awake 
stage. A new inhalational anesthetic, xenon has potential advantages for awake craniotomy because of its neuroprotective properties and rapid emergence [27].

\section{ANESTHETIC APPROACHES FOR AWAKE CRANIOTOMY: ANALGESIC COMPONENTS}

The mainstay of analgesia for awake craniotomy is a regional block, and there are two main approaches: the regional scalp block along with incision line infiltration, and the scalp nerve block for six nerves, which provide sensory innervation to the scalp [28]. Adequate regional blocks make the patient tolerable for the surgery and enables better patient satisfaction after surgery in terms of pain compared with general anesthesia. However, even when regional blocks are successfully performed, patients often experience and complain of pain during surgery. Usually, these sensations are associated with manipulations of the skull-base structures or traction of pain-sensitive intracranial structures [29]. Additional opioids may lead to poor cooperation of the patient and reduced reliability of brain mapping [6]. Adequate preoperative patient information and preparation, expert anesthesiologists, and surgical technique to avoid traction of pain-sensitive intracranial structures are the cornerstone to improve intraoperative pain management and ensure postoperative patient's satisfaction.

\section{COMPLICATIONS OF AWAKE CRANIOTOMY}

Seizures, hypertension, respiratory depression, nausea and vomiting, and mild brain swelling may occur during awake craniotomy [17]. Macroglossia [30] and accidental intracerebral injection of local anesthetics during scalp nerve block [31] have also been reported. Although complications during awake craniotomy are minor and easily manageable, urgent intervention should be performed for seizures and airway obstruction. A recent meta-analysis demonstrated that failure of awake craniotomy, defined as failure to achieve complete awake brain mapping occurs in $<2 \%$ cases, and the incidence of adverse events, such as seizures, conversion to general anesthesia, and new postoperative neurologic dysfunction, were similar between the AAA and MAC techniques [2].

The frequency of seizures during awake craniotomy was reported to vary between 2.9 and $54 \%$ in a multicenter retrospective study of 823 cases of intraoperative brain mapping [32]. The main risk factors for intraoperative seizure are history of preoperative seizure and frontal lobe tumors, and preoperative antiepileptic medication does not reduce the incidence of seizures [32,33]. Intraoperative seizures mainly occur due to electrical cortical stimulation during brain mapping, and the seizures can be easily controlled by cortical surface irrigation with cold saline by the surgeon. If this is ineffective, low doses of intravenous propofol can be administered [6]. The efficacy of antiepileptic drugs for intraoperative seizure prevention remains debatable. In a systematic review, antiepileptic drug prophylaxis did not show benefits of seizures prevention in brain tumor resection [34]. Another systematic review and meta-analysis reported that the efficacy of levetiracetam is superior to that of phenytoin and valproate in terms of seizure prevention [35]. However, evidence encouraging the routine use of antiepileptic drugs in awake craniotomy remains limited.

Airway obstruction is another serious complication encountered in awake craniotomy, but can be solved by simple intervention, such as jaw thrust and supplemental oxygen. In anticipation of airway emergencies, a full range of airway equipment including laryngeal mask airway, video laryngoscope, and endotracheal tube should be immediately available [6]. Moreover, it should be considered that laryngeal mask airway insertion, or endotracheal intubation are difficult in patients with semi-lateral position whose head is turned with a head frame.

\section{FUTURE CONSIDERATIONS FOR AWAKE CRANIOTOMY}

Awake craniotomy may be considered not only in brain tumor and epilepsy surgery, but also in other neurosurgical procedures, such as cerebral aneurysm clipping and excision of arteriovenous malformations [36,37].

Awake craniotomy has been performed to minimize the risk of motor and language function damage. However, an increasing interest is seen in the adoption of awake craniotomy for brain tumors located in the non-dominant hemisphere area, which is responsible for visuospatial and social cognition. Preservation of such functions is important especially for patients with low-grade glioma to return to their daily lives quickly after the surgery, and expect longer survival [38]. However, precise brain mapping of non-dominant hemisphere functions is particularly diffi- 
cult due to complexity of its functional anatomy. Therefore, it is necessary to develop adequate methods to assess large spectrum of cognition during awake brain surgery [39].

\section{CONCLUSION}

Anesthesia for awake craniotomy is one of the most challenging fields for anesthesiologists. Awake craniotomy is advantageous because it can perform extended range of tumor resection with less risk of neurological deficits including motor and speech function damage, compared with craniotomy under general anesthesia. Both the MAC and AAA techniques are feasible and safe anesthetic techniques for awake craniotomy, and adequate regional block is required for effective intraoperative pain control and better patient satisfaction. Appropriate patient selection, perioperative psychological support, and proper anesthetic management for individual patients in each stage of surgery are crucial for procedural safety, success, and patient satisfaction.

\section{CONFLICTS OF INTEREST}

No potential conflict of interest relevant to this article was reported.

\section{AUTHOR CONTRIBUTIONS}

Conceptualization: Seung Hyun Kim, Seung Ho Choi. Data acquisition: Seung Hyun Kim, Seung Ho Choi. Supervision: Seung Ho Choi. Writing-original draft: Seung Hyun Kim, Seung Ho Choi.

\section{ORCID}

Seung Hyun Kim, https://orcid.org/0000-0003-2127-6324

Seung Ho Choi, https://orcid.org/0000-0001-8442-4406

\section{REFERENCES}

1. Uff C, Frith D, Harrison C, Powell M, Kitchen N. Sir Victor Horsley's 19th century operations at the National Hospital for Neurology and Neurosurgery, Queen Square. J Neurosurg 2011; 114: 534-42.

2. Stevanovic A, Rossaint R, Veldeman M, Bilotta F, Coburn M. Anaesthesia management for awake craniotomy: systematic review and meta-analysis. PLoS One 2016; 11: e0156448.
3. Gerritsen JKW, Arends L, Klimek M, Dirven CMF, Vincent AJE. Impact of intraoperative stimulation mapping on high-grade glioma surgery outcome: a meta-analysis. Acta Neurochir (Wien) 2019; 161: 99-107.

4. Eseonu CI, Rincon-Torroella J, ReFaey K, Lee YM, Nangiana J, Vivas-Buitrago T, et al. Awake craniotomy vs craniotomy under general anesthesia for perirolandic gliomas: evaluating perioperative complications and extent of resection. Neurosurgery 2017; 81: 481-9

5. Meng L, Berger MS, Gelb AW. The potential benefits of awake craniotomy for brain tumor resection: an anesthesiologist's perspective. J Neurosurg Anesthesiol 2015; 27: 310-7.

6. Meng L, McDonagh DL, Berger MS, Gelb AW. Anesthesia for awake craniotomy: a how-to guide for the occasional practitioner. Can J Anaesth 2017; 64: 517-29.

7. Hervey-Jumper SL, Li J, Lau D, Molinaro AM, Perry DW, Meng L, et al. Awake craniotomy to maximize glioma resection: methods and technical nuances over a 27 -year period. J Neurosurg 2015; 123: 325-39.

8. Rajan S, Cata JP, Nada E, Weil R, Pal R, Avitsian R. Asleepawake-asleep craniotomy: a comparison with general anesthesia for resection of supratentorial tumors. J Clin Neurosci 2013; 20: 1068-73.

9. Bajwa SJ, Anand S, Kaur G. Anesthesia and cancer recurrences: the current knowledge and evidence. J Cancer Res Ther 2015; 11: $528-34$

10. Kim R. Effects of surgery and anesthetic choice on immunosuppression and cancer recurrence. J Transl Med 2018; 16: 8.

11. Meserve JR, Kaye AD, Prabhakar A, Urman RD. The role of analgesics in cancer propagation. Best Pract Res Clin Anaesthesiol 2014; 28: 139-51.

12. Brown T, Shah AH, Bregy A, Shah NH, Thambuswamy M, Barbarite $\mathrm{E}$, et al. Awake craniotomy for brain tumor resection: the rule rather than the exception? J Neurosurg Anesthesiol 2013; 25: $240-7$.

13. Richardson AM, McCarthy DJ, Sandhu J, Mayrand R, Guerrero C, Rosenberg C, et al. Predictors of successful discharge of patients on postoperative day 1 after craniotomy for brain tumor. World Neurosurg 2019; 126: e869-77.

14. Ruis C, Wajer IH, Robe P, van Zandvoort M. Anxiety in the preoperative phase of awake brain tumor surgery. Clin Neurol Neurosurg 2017; 157: 7-10.

15. Potters JW, Klimek M. Awake craniotomy: improving the patient's experience. Curr Opin Anaesthesiol 2015; 28: 511-6.

16. Skucas AP, Artru AA. Anesthetic complications of awake craniotomies for epilepsy surgery. Anesth Analg 2006; 102: 882-7.

17. Eseonu CI, ReFaey K, Garcia O, John A, Quiñones-Hinojosa A, 
Tripathi P. Awake craniotomy anesthesia: a comparison of the monitored anesthesia care and asleep-awake-asleep techniques. World Neurosurg 2017; 104: 679-86.

18. Dilmen OK, Akcil EF, Oguz A, Vehid H, Tunali Y. Comparison of conscious sedation and asleep-awake-asleep techniques for awake craniotomy. J Clin Neurosci 2017; 35: 30-4.

19. Kulikov A, Lubnin A. Anesthesia for awake craniotomy. Curr Opin Anaesthesiol 2018; 31: 506-10.

20. Sewell D, Smith M. Awake craniotomy: anesthetic considerations based on outcome evidence. Curr Opin Anaesthesiol 2019; 32: 546-52.

21. Lobo F, Beiras A. Propofol and remifentanil effect-site concentrations estimated by pharmacokinetic simulation and bispectral index monitoring during craniotomy with intraoperative awakening for brain tumor resection. J Neurosurg Anesthesiol 2007; 19: 183-9.

22. Soehle M, Wolf CF, Priston MJ, Neuloh G, Bien CG, Hoeft A, et al. Propofol pharmacodynamics and bispectral index during key moments of awake craniotomy. J Neurosurg Anesthesiol 2018; 30: 32-8.

23. Lin N, Vutskits L, Bebawy JF, Gelb AW. Perspectives on dexmedetomidine use for neurosurgical patients. J Neurosurg Anesthesiol 2019; 31: 366-77.

24. Goettel N, Bharadwaj S, Venkatraghavan L, Mehta J, Bernstein M, Manninen PH. Dexmedetomidine vs propofol-remifentanil conscious sedation for awake craniotomy: a prospective randomized controlled trial. Br J Anaesth 2016; 116: 811-21.

25. McAuliffe N, Nicholson S, Rigamonti A, Hare GMT, Cusimano M, Garavaglia M, et al. Awake craniotomy using dexmedetomidine and scalp blocks: a retrospective cohort study. Can J Anaesth 2018; 65: 1129-37.

26. Garavaglia MM, Das S, Cusimano MD, Crescini C, Mazer CD, Hare GM, et al. Anesthetic approach to high-risk patients and prolonged awake craniotomy using dexmedetomidine and scalp block. J Neurosurg Anesthesiol 2014; 26: 226-33.

27. Rylova A, Maze M. Protecting the brain with xenon anesthesia for neurosurgical procedures. J Neurosurg Anesthesiol 2019; 31: 18-29.

28. Potters JW, Klimek M. Local anesthetics for brain tumor resection: current perspectives. Local Reg Anesth 2018; 11: 1-8.
29. Fontaine D, Almairac F. Pain during awake craniotomy for brain tumor resection. Incidence, causes, consequences and management. Neurochirurgie 2017; 63: 204-7.

30. Cormack J, Karna S. Macroglossia during awake craniotomy: a near miss. J Neurosurg Anesthesiol 2018; 30: 383-4.

31. Lee WK, Kim H, Bae MI, Choi SH, Min KT. Accidental intracerebral injection and seizure during scalp nerve blocks for awake craniotomy in a previously craniotomized patient- a case report. Korean J Anesthesiol 2018; 71: 483-5.

32. Spena G, Schucht P, Seidel K, Rutten GJ, Freyschlag CF, D'Agata F, et al. Brain tumors in eloquent areas: a European multicenter survey of intraoperative mapping techniques, intraoperative seizures occurrence, and antiepileptic drug prophylaxis. Neurosurg Rev 2017; 40: 287-98.

33. Spena G, Roca E, Guerrini F, Panciani PP, Stanzani L, Salmaggi A, et al. Risk factors for intraoperative stimulation-related seizures during awake surgery: an analysis of 109 consecutive patients. J Neurooncol 2019; 145: 295-300.

34. Chandra V, Rock AK, Opalak C, Stary JM, Sima AP, Carr M, et al. A systematic review of perioperative seizure prophylaxis during brain tumor resection: the case for a multicenter randomized clinical trial. Neurosurg Focus 2017; 43: E18.

35. Pourzitaki C, Tsaousi G, Apostolidou E, Karakoulas K, Kouvelas D, Amaniti E. Efficacy and safety of prophylactic levetiracetam in supratentorial brain tumour surgery: a systematic review and meta-analysis. Br J Clin Pharmacol 2016; 82: 315-25.

36. Abdulrauf SI, Vuong P, Patel R, Sampath R, Ashour AM, Germany LM, et al. "Awake" clipping of cerebral aneurysms: report of initial series. J Neurosurg 2017; 127: 311-8.

37. Chan DYC, Chan DTM, Zhu CXL, Kan PKY, Ng AY, Hsieh YPS, et al. Awake craniotomy for excision of arteriovenous malformations? A qualitative comparison study with stereotactic radiosurgery. J Clin Neurosci 2018; 51: 52-6.

38. Duffau H. Is non-awake surgery for supratentorial adult lowgrade glioma treatment still feasible? Neurosurg Rev 2018; 41: 133-9.

39. Ruis C. Monitoring cognition during awake brain surgery in adults: a systematic review. J Clin Exp Neuropsychol 2018; 40: 1081-104. 